\title{
NECESIDAD SENTIDA DE LAS MUJERES SORDAS DURANTE EL PARTO Y EL PUERPERIO INMEDIATO EN EL ÁMBITO HOSPITALARIO
}

\author{
Flavia Polanco Teijo *, Salvador García-Ruise ** \\ *Enfermera, Residente de Enfermería Obstétrico-Ginecológica. \\ **Residente de Medicina Preventiva y Salud Pública.
}

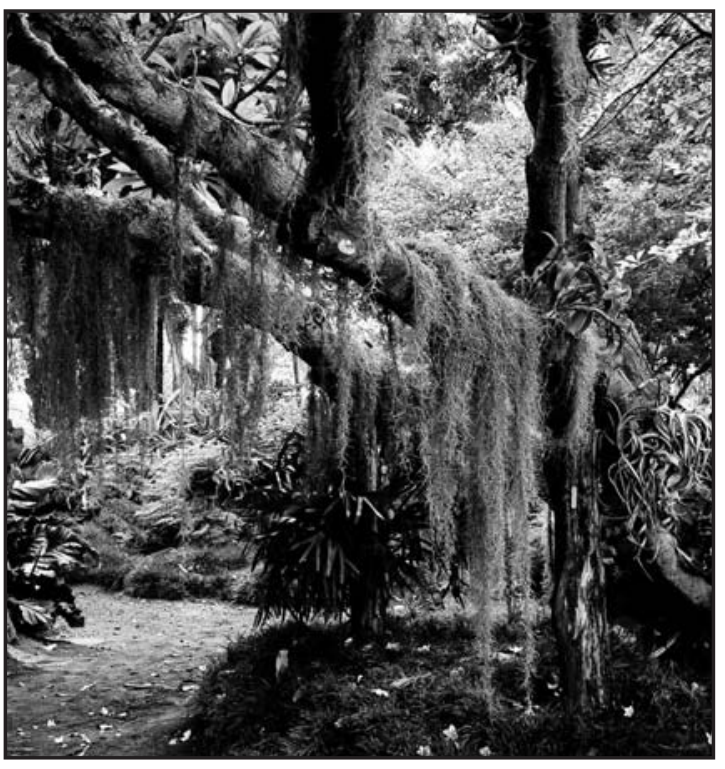

FELT NEED OF DEAF WOMEN DURING CHILDBIRTH AND IMMEDIATE POSTPARTUM IN THE HOSPITAL

\section{SUMMARY}

Introduction: Estimates by the National Statistics Institute (INE), as data referring to 2008, in Spain there are 40,000 Deaf women on fertile age (16-44 years).

The maternity in the women with disability has a cost extra in relation to the rest of women. Also tend to be unemployed or have works of minor qualification.

Objective: To know and understand the perceived need for both Deaf women in childbirth and in the puerperium.

Methods: Qualitative study from semi-structured interviews with Deaf women in the immediate postpartum period in the area of University Hospital of Santiago de Compostela.

Results: Deaf patients use health services differently than the listeners; they usually have problems such as fear, distrust and frustration. They showed difficulties in communicating with health personnel during their hospital admission, both verbal and nonverbal language. The lack of information was also common in all of them, mostly during the procedures and techniques which were performed.

Conclusions: Deaf women and health personnel have communication difficulties involving the establishment of links that are required in health care and that may adversely affect the diagnosis and treatment.

Key words: Deaf woman, health care, childbirth, puerperium.

ENFERMAGEM, TERAPIAS COMPLEMENTARES E ALTERNATIVAS. JUSTIFICAÇÃO ANTROPOLÓGICA DO ESTUDO.

\section{RESUMO}

Introdução: Segundo estimativas do Instituto Nacional de Estatística (INE), na Espanha, 40.000 mulheres são surdas em idade fértil (16 a 44 anos).

A maternidade nas mulheres com incapacidade tem um custo de oportunidade extra em relação a outras mulheres. Ademais, essa situação pode favorecer para que estejam desempregadas ou tenham trabalhos de menor qualificação.

Objetivo: Conhecer e comprender a necessidade 
sentida pelas mulheres surdas no parto e puerperio imediato.

Material e métodos: Estudo qualitativo realizado a partir de entrevistas semiestruturadas com mulheres surdas durante o puerperio imediato no âmbito do Complejo Hospitalario Universitario de Santiago de Compostela (CHUS).

Resultados: As pacientes surdas utilizam os serviços de saúde de forma distinta daquelas que ouvem; manifestam dificuldades como medo, desconfiança e frustrações. Mostraram problemas na comunicação com o pessoal de saúde durante seu ingresso no hospital, tanto na linguagem verbal como na não verbal. A falta de informação foi também uma dificuldade comum em tidas elas, principalmente durante os procedimentos e técnicas realizadas.

Conclusões: Os resultados deste estudo mostram que as mulheres surdas e o pessoal de saúde têm dificuldade de comunicação que comprometem o estabelecimento de vínculos necessários na atenção à saúde e que podem afetar negativamente o diagnóstico e o tratamento.

Palavras chave: Mulher Surda, atenção à saúde, parto, puerpério.

\section{RESUMEN}

Introducción: Según estimaciones del Instituto Nacional de Estadística (INE), según datos referidos al año 2008, en España hay 40.000 mujeres Sordas en edad fértil (16-44 años).

La maternidad en las mujeres con discapacidad tiene un coste extra en relación al resto de mujeres. Además suelen estar desempleadas o tener trabajos de menor cualificación.

Objetivo: Conocer y comprender la necesidad sentida de las mujeres Sordas en el parto y puerperio inmediato.

Material y método: Estudio cualitativo etnográfico a partir de entrevistas semiestructuradas a mujeres Sordas durante el puerperio inmediato en el ámbito del Complejo Hospitalario Universitario de Santiago de Compostela (CHUS).

Resultados: Las pacientes Sordas utilizan los servicios sanitarios de forma distinta que los oyentes; manifiestan dificultades como miedo, desconfianza y frustraciones. Mostraron problemas en la comunicación con el personal sanitario durante su ingreso hospitalario, tanto en el lenguaje verbal como en el no verbal. La falta de información fue también una dificultad común en todas ellas, principalmente durante los procedimientos y técnicas que se les realizaron.

Conclusiones: Los resultados de este estudio muestran que las mujeres Sordas y el personal sanitario tienen dificultades de comunicación que comprometen el establecimiento de vínculos necesarios en la atención sanitaria y que pueden afectar negativamente al diagnóstico y tratamiento.

Palabras clave: Mujer Sorda, atención sanitaria, parto, puerperio.

\section{INTRODUCCIÓN}

La World Federation of the Deaf (WFD) define a la persona Sorda como "aquella que usa la lengua de signos como modo de comunicación primario, se identifica a sí misma con otras personas Sordas y usualmente no oye" (Xunta de Galicia, 2003).

Según las estimaciones de la Organización Mundial de la Salud (OMS), en el año 2005 presentaban una pérdida de audición severa en ambos oídos 278 millones de personas en todo el mundo, y se estima que la prevalencia de hipoacusia severa o profunda en el neonato es del 1 por mil, que se eleva al 5 por mil cuando incluimos todos los grados de hipoacusia (Jokinen, 2010).

En España, cada año nacen alrededor de 2.000 niños con problemas auditivos, de los cuales la mitad presentan una sordera neurosensorial bilateral profunda. El $80 \%$ de las sorderas infantiles están presentes en el momento del nacimiento (Confederación Española de padres y amigos de los Sordos, 2004).

En España hay más de un millón de personas Sordas (Instituto Nacional de Estadística, 2008) de 


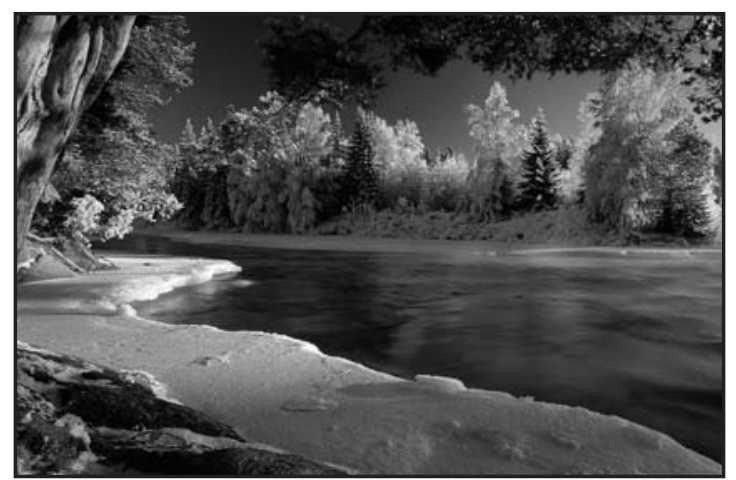

la cuales 40.000 son mujeres en edad fértil (16-44 años). (Tabla 1)

En Galicia existen 84.900 personas de 6 y más años, de ambos sexos, con discapacidad auditiva (Instituto Nacional de Estadística, 2008) de las cuales 3.200 son mujeres entre 6 y 44 años. (Tabla 2)

Según la Encuesta sobre Discapacidades, Autonomía personal y situaciones de Dependencia realizada por el Instituto Nacional de Estadística (INE) en el año 2008, sólo un 31\% del total de la población Sorda tenía Certificado de Minusvalía por deficiencias del oído (Instituto Nacional de Estadística, 2008). Algunas posibles causas de esta discordancia pueden ser la falta de aceptación de la sordera por parte de muchas personas y la negativa a obtener un certificado que estigmatiza como "minusválido", el desconocimiento de su utilidad y de las ventajas legales que comporta, el temor a la dificultad de encontrar un trabajo, etc. (Esteban, 2004).

\section{Modelo socio-cultural de la sordera}

Desde el punto de vista cultural, la sordera es un fenómeno socio-cultural y se considera a las personas Sordas como un grupo social minoritario con una lengua, una historia y una cultura propias (Minguet, 2000). El uso de la palabra 'sordo' en minúsculas hace referencia a una característica física (incapacidad física para oír), mientras que el término 'Sordo' en mayúsculas se usa habitualmente para referirse a los aspectos culturales y lingüísticos ligados a la condición de ser sordo (Minguet, 2000).

Dentro de la discapacidad auditiva existen distintos grados (según la intensidad o el comienzo de la sordera) que pueden dar lugar a diferentes nece- sidades sociales e influyen en la identidad que asumen las personas afectadas (Minguet, 2000; Muñoz \& Ruiz, 2000).

La sordera sigue ocupando actualmente el segundo puesto a nivel cuantitativo entre los déficits sensoriales. Suele darse con alta incidencia en la primera infancia, pasando desapercibida en los casos de sorderas leves y moderadas, lo que provoca retrasos cognitivos-lingüísticos graves en muchos casos (Losada, 1999).

Hay una enorme desigualdad educativa que afecta a las personas Sordas respecto al resto de la población. Esto condiciona que las personas Sordas tengan unos niveles de desarrollo social y educativo inferiores a los niveles medios que alcanza el resto de la población, lo que da lugar a numerosas y graves situaciones de exclusión social (Esteban, 2004).

Muchas de las personas Sordas que han terminado los estudios primarios sufren analfabetismo funcional o tienen serias dificultades para comprender textos y para expresarse correctamente por escrito. Se observa también que la desigualdad educativa la sufren en mayor medida las mujeres Sordas respecto a los varones (Esteban, 2004). (Tabla 3)

En el caso de las mujeres Sordas, el 67,2\% de ellas están en situación de inactividad económica, y la tasa de paro afecta a casi el $30 \%$ de la población activa femenina (Esteban, 2004). (Tabla 4)

El $60 \%$ de las mujeres Sordas que se encuentran en esta situación se dedican a labores del hogar, lo que confirma que es el colectivo que se encuentra socialmente más excluido (Esteban, 2004).

\section{Maternidad y discapacidad}

Existen tres factores que influyen en el gasto extraordinario que una mujer con discapacidad auditiva debe realizar para mantener el mismo nivel de vida que otra sin discapacidad en las mismas circunstancias para el desarrollo de su maternidad. Estos son (Iniesta, 2004):

El nivel de necesidad extraordinaria como resultado de su discapacidad (adquisición de avisadores visuales, fax, etc.).

La dependencia del nivel de provisión de servicios sociales, beneficios de la seguridad social, 
prestaciones económicas, y exenciones fiscales existentes para cubrir esta necesidad específica derivada de la confluencia de la discapacidad y la maternidad.

El efecto de la discapacidad y la maternidad en los ingresos (coste de oportunidad).

La maternidad en las mujeres con discapacidad tiene un coste extra con respecto a otras mujeres. Las madres con discapacidad suelen tener ingresos inferiores a los de otras mujeres o personas sin discapacidad, y además cuando tienen un hijo la conciliación de la vida familiar y laboral se hace más difícil. Además, las madres con discapacidad suelen estar desempleadas o tener trabajos de menor cualificación (Pélaez, Martínez \& Leonhardt, 2009).

Numerosos estudios evidencian la existencia de barreras de comunicación entre el paciente Sordo y los profesionales de la salud (Chaveiro, Celeno \& Alves, 2009; Harmer, 1999). Debido a esto las personas Sordas o hipoacúsicas reciben una atención médica a menudo inadecuada e inapropiada.

Desde la perspectiva de la integración en la sociedad oyente a través de la igualdad, de la normalización de los servicios, en este trabajo se analizan e interpretan los problemas de las mujeres Sordas durante el parto y el puerperio inmediato en el ámbito hospitalario. El objetivo de este trabajo es conocer y comprender la necesidad sentida de las mujeres Sordas tanto en el parto como en el puerperio inmediato.

\section{MATERIAL Y MÉTODO}

Se ha realizado un estudio cualitativo etnográfico a partir de entrevistas semiestructuradas a mujeres Sordas en el puerperio inmediato en el ámbito del Complejo Hospitalario Universitario de Santiago de Compostela (CHUS).

El período de estudio fue el comprendido entre Enero y Mayo de 2010.

Las participantes debían ser mujeres Sordas que ingresaran por parto y/o permanecieran en el hospital durante el puerperio inmediato y emplearan el lenguaje de signos como medio principal de comunicación. Como criterio de exclusión se estableció la negativa de la paciente a participar en este estudio.

Se adoptó la forma del consentimiento verbal para la participación en el estudio, garantizando la confidencialidad de la información obtenida y protegiendo su intimidad.

Las entrevistas se realizaron durante el puerperio inmediato en las habitaciones de las pacientes en la planta de Obstetricia el día posterior al parto, con una duración media de 30 minutos.

El grupo de investigación estaba integrado por una residente de enfermería obstétrico-ginecológica y un intérprete de LSE.

La entrevista constaba de varios apartados que incluían preguntas sobre variables sociodemográficas (edad, nivel de estudios, profesión), datos obstétricos $\left(\mathrm{n}^{\mathrm{o}}\right.$ gestaciones, semanas amenorrea, empleo de analgesia epidural y realización o no de episiotomía) y su experiencia/opinión personal sobre las revisiones del embarazo en la matrona y el ginecólogo, las clases de preparación al parto, ingresos hospitalarios previos, el momento del parto y el puerperio. A sus familiares se les preguntaba sobre su experiencia y visión del nacimiento.

\section{RESULTADOS}

Se entrevistaron tres mujeres Sordas puérperas y sus familiares.

La edad media fue de $30 \pm 2,66$ años y los partos fueron a término con una media de $39 \pm 0,75$ semanas de amenorrea. Las tres mujeres solicitaron analgesia epidural y a dos de ellas se les realizó episiotomía.

Con respecto al nivel de estudios, todas ellas tenían estudios básicos o primarios, pero sólo una trabajaba (de limpiadora), dedicándose el resto a las labores del hogar. Con respecto a sus parejas, uno tenía estudios secundarios y los otros dos estudios primarios, trabajando en el sector servicios. Dos de ellos eran Sordos y uno oyente.

A la pregunta ¿Acudía a las revisiones de la matrona y el ginecólogo acompañada de alguien? ¿De quién?, las respuestas de las tres mujeres coincidían:

“...Para las revisiones, siempre tengo que ir acompañada de mi madre o de un familiar, porque no me gusta que un intérprete esté presente en la consulta. Pero es mi madre,... y no quiero que sepa algunas cosas, y además no sabe muchas palabras en lengua de signos y no se expresa bien...". La madre de esta puérpera es oyente. 
Cuando se les pregunta si habían acudido a clases de preparación al parto, contestan que no, explicando sus motivos:

“...No tuve una preparación para el parto, porque iba a las clases con una intérprete y mi esposo, y nos resultaban tan aburridas que los tres nos poníamos a charlar y no me enteraba de lo que se decía. Era tan aburrido que dejamos de ir tras una o dos clases". "...Si hubiera tenido una matrona con la que comunicarme directamente, a la que preguntarle mis dudas, y que me diera instrucciones que yo pudiera entender, claro que hubiera asistido a todas las clases...".

Cuando se les preguntaba por su experiencia personal en ingresos hospitalarios anteriores, sólo dos de ellas habían estado ingresadas:

“...He tenido otros ingresos (hospitalarios) previos, y tuve algunos problemas por confusiones en la medicación. Por ello ahora, cuando ingreso en el hospital, tengo mucho miedo a las equivocaciones, porque no puedo entenderme con nadie cuando preguntan mi nombre..."

"...Una vez cuando estuve ingresada me pusieron la morfina de una compañera de habitación, y estuve durmiendo horas y horas..."

“...Recién operada de una apendicitis, en la sala de recuperación, me dieron una comida normal porque no les pude decir mi nombre ni explicarles que estaba recién operada, yo creía que era la costumbre, comer normal tras una operación. Ahora procuro tener a algún familiar oyente siempre conmigo...".

"...Cuando ingreso en el hospital, no quiero quedarme sola de noche por miedo a que me confundan con otro paciente, o de tener que llamar a la enfermera y no poder explicarle lo que me ha ocurrido, o de que vengan a preguntarme algo y no pueda explicarlo...".

“...Cuando estuve ingresada los médicos pasan visita, echan a la familia, y yo me quedo incomunicada. Algunas veces mi madre conseguía quedarse, pero por lo general no tengo forma de comunicarme con el médico que me está tratando, ni de saber cómo voy o lo que está pasando, ni entender las explicaciones del personal sobre lo el curso de mi enfermedad...".

Al preguntarles cuál ha sido su experiencia con el personal sanitario durante el ingreso hospitala- rio, responden:

"...No puedo entenderme con nadie, ni explicarles lo que me duele o lo que tengo, ni entender el tratamiento o lo que tengo que hacer. No hay forma de entenderme con nadie cuando estoy enferma...".

“...Todo el mundo me habla a gritos, yo no puedo oír nada, pero en lugar de hacerme algún gesto o lo que sea, me gritan, y no me entero absolutamente de nada...".

En el momento del parto, todas ellas señalan lo importante que era para ellas el contacto visual:

“...Durante el parto, me sentía muy angustiada porque a ratos no veía la cara de la matrona y sentía que no podía comunicarme con ella, ni recibir sus instrucciones ni comunicarle cuándo tenía contracciones. Intentaba que me mirase a la cara todo el tiempo y cuando eso no era posible lo pasaba fatal...".

“...Durante todo el parto, aunque las matronas se han portado muy bien conmigo y me han explicado lo que han podido a través de la lectura labial, necesitaba saber lo que estaba pasando, lo que tenía que hacer, si empujar o no con las contracciones, o lo que me iban a hacer...".

Sin embargo, todas ellas señalan como una necesidad común la falta de información durante todo su embarazo:

“...Lo que más eché en falta fue la falta de información...”.

Respecto a sus familiares, los acompañantes Sordos coincidían en señalar la falta de medios visuales de aviso en el hospital:

“...Durante las esperas en el paritorio, mi esposo (que también es Sordo) lo pasó muy mal porque llamaban a los familiares para entrar al paritorio mediante un teléfono que hay en la sala de espera, y él le iba diciendo a todo el que había cerca que por favor lo avisasen si llamaban a alguien que no estuviese, porque él no podía oír cuando lo llamaran...".

\section{DISCUSIÓN}

Las mujeres Sordas entrevistadas usan los servicios sanitarios de forma distinta que las mujeres oyentes; manifiestan dificultades como miedo, desconfianza y frustraciones (Barnett, 2002).

La comunicación efectiva con los pacientes 
Sordos es importante en el cuidado de la salud, ya que una comunicación inadecuada puede llevar a diagnósticos erróneos y tratamientos equivocados (Meador \& Zazove, 2005); esta comunicación sigue siendo descuidada en los sistemas de salud (McAleer, 2005), a lo que se suma la falta de capacidad de comunicación no verbal por parte del personal sanitario (McAleer, 2005). El lenguaje no verbal es una forma de comunicación que necesita ser entendido y validado en los servicios de salud. Es esencial para poder interpretar aspectos tales como gestos o expresiones faciales y/o corporales (Chaveiro, Barbosa \& Porto, 2008; Lezzoni, O’Day, Killeen \& Harker, 2004).

En la prestación de la asistencia sanitaria, las mujeres Sordas entrevistadas manifestaron que no se tuvieron en cuenta aspectos culturales, lingüísticos, sociológicos, psicológicos y educativos de su discapacidad auditiva (Muñoz \& Ruiz, 2000).

Una necesidad común en todas las entrevistadas era la información, que es uno de los principales obstáculos a los que se enfrentan diariamente las personas Sordas (Steinberg, Barnett, Meador, Wiggins \& Zazove, 2006).

Son frecuentes las dificultades que estas pacientes tienen para entender regímenes terapéuticos, dosis de medicación o sus efectos secundarios (Meador \& Zazove, 2005).

Sin intérpretes de LSE o acompañantes oyentes, las pacientes no son conscientes de información crucial, precipitando emociones desde la frustración al terror (Barnett, 2002).

La ausencia de intimidad en las consultas es un hecho reconocido por las entrevistadas, y viven con resignación la falta de independencia y autonomía, ya que deben de ir acompañadas de otros familiares o intérpretes (Lezzoni, O’Day, Killeen \& Harker, 2004).

Las mujeres entrevistadas ponen de manifiesto la existencia de barreras de comunicación al no disponer en servicios sanitarios de video-teléfonos, teléfonos de texto, timbres con señales luminosas,... (Steinberg, Barnett, Meador, Wiggins \& Zazove, 2006). El hecho de que la sordera sea una minusvalía invisible ha derivado en que la sensibilización hacia las barreras de comunicación pase desapercibida como tal.

\section{CONCLUSIONES:}

Este estudio muestra que las mujeres Sordas durante el parto y el puerperio inmediato en el ámbito hospitalario y el personal sanitario presentan dificultades de comunicación. Estas dificultades comprometen el establecimiento de vínculos necesarios en la atención sanitaria que pueden afectar negativamente al diagnóstico y al tratamiento.

Hay una necesidad innegable de mejorar la comunicación entre el personal sanitario y los pacientes Sordos. Para solventar las barreras de comunicación estructurales es preciso intentar que la información y la comunicación social sean difundidas por medios visuales como imágenes, iconos o lenguaje escrito.

El problema que se plantea para las personas Sordas en el acceso a servicios sanitarios, ya sean públicos o privados, tiene difícil solución, y pueden necesitar la ayuda de un intérprete de LSE para eliminar las barreras de comunicación interpersonales. El acceso a este recurso puede ser complejo por diversos motivos:

- El escaso número de intérpretes de LSE hace que puedan aparecer dificultades para solicitar sus servicios.

- En situaciones de urgencia, como es un ingreso hospitalario, puede ser difícil localizar a un intérprete de LSE.

- La distorsión en la emisión y recepción de los mensajes.

- En la traducción de un mensaje de una persona Sorda por un intérprete, se pierde parte del mismo porque no se tiene en cuenta la comunicación no verbal, los sentimientos,... Además, las estructuras sintácticas de la lengua de signos son completamente diferentes de la expresión verbal, lo que añade dificultad al acto de la interpretación o traducción.

- La falta de intimidad.

- Esta falta de intimidad se ve incrementada porque los intérpretes de LSE pertenecen con frecuencia al ámbito de la comunidad de Sordos.

En cuanto a los aspectos antropológicos de los cuidados, uno de los factores más importantes que afecta a la calidad y la adecuación de la atención 
prestada a los pacientes Sordos es la falta de sensibilización de los trabajadores de la salud acerca de las personas Sordas.

Es necesario conocer las características de la identidad y la cultura Sordas a fin de facilitar habilidades de comunicación y mejorar la relación entre el paciente Sordo y el personal sanitario.

Comprender los problemas que rodean la asistencia sanitaria de las personas Sordas facilitará la interacción entre estos pacientes y el personal sanitario. Se debe tener en cuenta que las personas Sordas están capacitadas para decidir por sí mismas en temas de salud y participar en su asistencia sanitaria.

Los derechos de acceso al sistema sanitario de las personas Sordas deben ser garantizados impartiendo una formación adecuada al personal sanitario para atender a este segmento de la población, destruyendo las barreras de comunicación que nacen de la incomprensión e incluso de la negación de la realidad del otro.

\section{BIBLIOGRAFÍA}

- Barnett S. (2002). Communication with deaf and hard-of-hearing people: A guide for medical education. Academic Medicine, 77, 694-700.

- Chan M. (2010). Organización Mundial de la Salud. Recuperado el 20 de Mayo de 2010, de http://www.who.int/es/

- Chaveiro N, Barbosa MA, Porto CC. (2008). Literature revision about the attendance of deaf patient by health professionals. Revista da Escola de Enfermagen, 42, 578-83.

- Chaveiro N, Celeno C, Alves M. (2009). The relation between deaf patients and the doctor. Brazilian Journal of Otorhinolaryngology, 75, 147-50.

- Confederación Española de padres y amigos de los Sordos. (2004). Manual básico de formación especializada sobre discapacidad auditiva. Madrid: FIAPAS.
- Esteban Saiz ML. Coordinador. (2004). Libro blanco de la Lengua de Signos Española en el Sistema Educativo. Madrid: Confederación Estatal de Personas Sordas.

- Harmer L. (1999). Health care delivery and deaf people: practice, problems, and recommendations for change. Journal of Deaf Studies and Deaf Education, 4, 73-110.

- Iniesta Martínez A. (2004). La mujer sorda en la vida privada y pública. Alicante: Universidad de Alicante.

- Instituto Nacional de Estadística. (2008). Encuesta sobre Discapacidades, Autonomía personal y situaciones de Dependencia. Madrid: Instituto Nacional de Estadística.

- Jokinen M. (2010). World Federation of the Deaf. Recuperado el 10 de Abril de 2010, de http://www.wfdeaf.org/default.aspx

- Lezzoni L, O'Day B, Killeen M, Harker H. (2004). Commnicating about health care: observations from persons who area deaf or hard of hearing. American College of Physicians, 140, 356-63.

- Losada Martínez B. (1999). Modelos actuales en educación de sordos: lengua de signos, bilingüismo e integración. Tesis no publicada, Departamento de Psicología evolutiva y de la educación, Universidad de Santiago de Compostela.

- McAleer M. (2005). Communicating effectively with deaf patients. Nursing Standard, 20, 51-54.

- Meador HE, Zazove P. (2005). Health care interactions with deaf culture. Journal of the American Board of Family Practice, 18, 218-22.

- Minguet Soto A. coordinador. (2000). Rasgos sociológicos y culturales de las personas Sordas. Valencia: Federación de Sordos de la Comunidad Valenciana.

- Muñoz I, Ruiz M T. (2000). Empowering the deaf: let the deaf be deaf. Journal of Epidemiology and Community Health, 54, 40-4.

- Pélaez A, Martínez B, Leonhardt M. (2009). Maternidad y discapacidad. Madrid: Cinca.

- Steinberg AG, Barnett S, Meador HE, Wiggins EA, Zazove P. (2006). Health care system accessibility. Experiences and perceptions of deaf people. Journal of General Internal Medicine, 21, 260-6.

- Xunta de Galicia. (2003). Guía para la eliminación de las barreras de comunicación para personas Sordas y con discapacidad auditiva. Santiago de Compostela: Consellería Sanidade.

\section{Tabla 1}

\begin{tabular}{|c|c|c|c|}
\hline España & $\begin{array}{c}\text { De } 16 \text { a } 24 \\
\text { años }\end{array}$ & $\begin{array}{c}\text { De } 25 \text { a 34 } \\
\text { años }\end{array}$ & $\begin{array}{c}\text { De 35 a } 44 \\
\text { años }\end{array}$ \\
\hline Mujeres & 4,4 & 10,7 & 24,3 \\
\hline
\end{tabular}

Tabla 1.Mujeres Sordas por grupos de edad (por mil habitantes). España.

Fuente: INE. Encuesta sobre Discapacidades, Autonomía personal y situaciones de Dependencia 2008 
Tabla 2

\begin{tabular}{|c|c|c|c|c|}
\hline Galicia & De 6 a 44 años & $\begin{array}{c}\text { De } 45 \text { a } 64 \\
\text { años }\end{array}$ & $\begin{array}{c}\text { De } 65 \text { a } 79 \\
\text { años }\end{array}$ & $\begin{array}{c}\text { De } 80 \text { y más } \\
\text { años }\end{array}$ \\
\hline Mujeres & 3,2 & 5,7 & 17,2 & 24,9 \\
\hline
\end{tabular}

Tabla 2. Mujeres Sordas por grupos de edad (por mil habitantes). Galicia.

Fuente: INE. Encuesta sobre Discapacidades, Autonomía personal y situaciones de Dependencia 2008

Tabla 3

\begin{tabular}{|c|c|c|}
\hline Nimed de estudios & $\begin{array}{l}\text { Mujeres } 10 y \\
\text { mass anos }\end{array}$ & $\begin{array}{l}\text { Varones } 10 \text { y más } \\
\text { anos }\end{array}$ \\
\hline Aralliabetor & 15.45 & 7 \\
\hline Sin estudios & 36,7 & 33,31 \\
\hline Estudios Primarios y ESO & 42.15 & 48,68 \\
\hline $\begin{array}{l}\text { Enseñanza Secundaria y Eroeciarca } \\
\text { Profesional de Primer y Segundo Grado }\end{array}$ & 3,18 & 5,48 \\
\hline Ensenanzas profesionales superiores & 0,43 & 1,63 \\
\hline \multirow[t]{2}{*}{ Estudios universicarios y equivientes } & 2,05 & 3,86 \\
\hline & 100 & 100 \\
\hline
\end{tabular}

Tabla 3. Distribución porcentual de personas sordas de 10 y más años por nivel de estudios terminados y sexo.

Fuente: Libro blanco de la Lengua de Signos Española en el Sistema Educativo. 2004

Tabla 4

\begin{tabular}{|l|c|c|c|}
\hline & Tasa de actividad & Tasa de inactividad & Tasa de desempleo \\
\hline Ambos sexos & 45.41 & 54,58 & 19,75 \\
\hline Varones & 57.19 & 42.80 & 14.99 \\
\hline Mujeres & 32.80 & 67,19 & 28,65 \\
\hline
\end{tabular}

Tabla 4. Porcentaje de personas sordas por sexos según relación con la actividad económica Fuente: Libro blanco de la Lengua de Signos Española en el Sistema Educativo. 2004 